\title{
PROMOCIÓN DE LA SALUD MENTAL: CO-PRODUCCIÓN DE SPOT SONOROS VINCULANDO LA UNIVERSIDAD CON LA COMUNIDAD
}

\author{
PROMOTING MENTAL HEALTH: CO-PRODUCTION OF \\ RADIO ADVERTISEMENTS LINKING UNIVERSITY AND \\ COMMUNITY
}

\author{
Sandra Ines Cerino* \\ Patricia Fabiana Gomez** \\ Marìa Cristina Cometto*** \\ Silvia Catalini*** \\ Marcela Alejandra Coronado ${ }^{* * * * *}$ \\ Julio Cesar Torres ${ }^{* * * * * * *}$ \\ Susana Sanguineti \\ Marta Pereyra*
}

\section{RESUMEN:}

El presente artículo comunica la experiencia extensionista de "Promoción de la salud mental en producción de spots sonoros" centrado en la relación comunicación/salud para fortalecer el derecho de la ciudadanía para mejorar su calidad de vida. Participaron estudiantes y equipos de cátedra de tres unidades académicas de dos universidades: Universidad Nacional de Córdoba y Universidad Siglo 21. Objetivo: producir y realizar interdisciplinariamente piezas sonoras para una campaña de promoción y prevención de salud mental. Destinatarios: población cordobesa. Metodología: relato de experiencia. Desarrollo: siete etapas simultáneas al dictado de las materias, utilizando la pedagogía crítica. Resultados: el $100 \%$ de los grupos lograron el objetivo, produciéndose 40 spot sonoros. Logros: participación familiares y amigos de los estudiantes para la grabación de los spot, facilitación del aprendizaje grupal, difusión de los resultados a radios de otras provincias. Dificultad: lograr acuerdos, tolerar, ceder, argumentar y equilibrar el esfuerzo de trabajo.

Palavras-chave: Promoción de la salud; Salud mental; Salud pública; Recursos audiovisuales.

\begin{abstract}
:
This article reports the university extension experience "Promotion of mental health through the production of advertisements focusing the communication/health relationship to strengthen the right of citizens to improve their quality of life. Students and professors from three academic units belonging to two universities took part in the project: Universidad Nacional de Córdoba and Universidad Siglo 21. Objective: to produce and carry out interdisciplinary radio advertisements for a campaign of promotion and prevention of mental health Targets: population of Cordoba. Methodology: experience report. Development: seven stages simultaneously with the teaching of subjects, using critical pedagogy. Results: all the groups achieved the objective, producing 40 advertisements. Achievements: participation of family members and friends of students for the recording of the advertisements, promotion of interdisciplinary work and group learning, dissemination of the results to radio stations in other provinces. Difficulty: reaching agreements, tolerating, giving in, discussing and balancing the work effort.
\end{abstract}

Keywords: Health promotion; Mental health; Public health; Audiovisual aids. 


\section{Introducción}

"En campaña" surgió como iniciativa en el año 2015 para mejorar la vinculación y divulgación de temáticas sensibles hacia la comunidad. Nuevamente este año, se inicia la experiencia extensionista para articular el campo de la comunicación con el de la salud mental, cuyo objetivo es producir y realizar desde la interdisciplina piezas sonoras de una campaña radiofónica de promoción y prevención de salud mental destinada a la población cordobesa. En esta oportunidad participaron tres unidades académicas y dos universidades: Cátedra de Producción Radial de la Carrera de Publicidad, Universidad Siglo 21; Cátedra de Producción Radiofónica de la Facultad de Ciencias de la Comunicación y Cátedra de Enfermería en Salud Mental y Psiquiatría, Escuela de Enfermería, Facultad de Ciencias Médicas, ambas de la Universidad Nacional de Córdoba.

El desarrollo de la experiencia se enfocó desde la pedagogía crítica y reflexiva, centrándose en una puesta en común y el aprendizaje colectivo y significativo, de modo que cada participante se enriqueció con lo aportado por los otros (pertenecientes o no a la misma unidad académica). La interdisciplinariedad es, así, una experiencia de vinculación entre cátedras, permite innovaciones pedagógicas para la promoción de la salud y desarrollo comunitario, fortalece el vínculo entre la universidad y la comunidad, creando una herramienta para facilitar la comunicación sobre la salud, desde una perspectiva que respeta los derechos de las personas vinculadas directas o indirectamente con la temática.

"En campaña" es la experiencia que tiene como antecedentes el trabajo conjunto de las cátedras mencionadas en otras actividades extensionistas centradas en la articulación entre la salud mental y los medios de comunicación (Pereyra, 2015b). Su sustento teórico está dado por la ley nacional de salud mental y las recomendaciones internacionales para el aporte de los medios de comunicación a la protección y promoción de la salud de las poblaciones. En Argentina, la Ley Nacional de Salud Mental acentúa la importancia de implementar prácticas de prevención y promoción de salud mental, basado en la intersectorialidad y el trabajo interdisciplinario para acercar a las poblaciones el acceso a la salud. (ARGENTINA. Poder Legislativo Nacional, 2010).

Conjuntamente la ley de Servicios de Comunicación Audiovisual (ARGENTINA. Presidencia de la Nación. Defensoria del PÚBLICO, 2019) y las recomendaciones para el tratamiento en los medios de temas de salud mental (argentina. presidencia de la nación. ministerio de salud, 2019; AUTORIDAD FEDERAL DE SERVICIOS DE COMUNICACIÓN AUDIOVISUA, 2010; GUÍA para el tratamiento mediático..., 2014; ARGENTINA. Ministerio de Salud. Dirección Nacional de Salud Mental y Adicciones, 2019) promueven el derecho a la comunicación y destaca que los medios deben evitar la discriminación basada en la presencia de discapacidades, velar por la normativa existente en materia de salud y procurar el respeto de los derechos en los medios. En sus principios $\mathrm{y}$, a través de su articulado, la Ley impulsa también la posibilidad de que todas las personas y sectores sociales puedan expresarse a través de los medios de comunicación audiovisual.
Este sentido comunitario, que incluye diversos actores, recupera el vínculo entre prácticas comunitarias, salud mental, sistema de salud y docencia que invitan a revisar los marcos teóricos hacia el reconocimiento de la salud y el sufrimiento psíquico como procesos sociales complejos que requieren de diversas modalidades de abordaje. (BANG, 2014b, 2014c). Así se reconocen múltiples determinantes y entrecruzamientos en el campo de problemáticas de salud/salud mental, su abordaje incluye, necesariamente, un enfoque comunitario, complejo e integral, siendo la protección de derechos una estrategia fundamental. (BANG, 2014a; VAZQUEZ, 2015).

En el marco de este proyecto se considera la salud mental colectiva como fundamento de la propuesta, pues hace referencia a la idea de promoción de la salud asociada a un conjunto de valores colectivos: vida, salud, solidaridad, equidad, democracia, ciudadanía, desarrollo, participación y asociación, entre otros. (BANG, 2014b). Estos conceptos son recuperados por los alumnos para fortalecer la capacidad colectiva de protección de una salud mental positiva y mejorar la calidad de vida. (ORGANIZACION MUNDIAL SALUD, 2018).

Consecuentemente, las acciones de promoción de salud mental comunitaria (HENAO et al., 2016) son aquellas que propician la transformación de los lazos comunitarios hacia vínculos solidarios y la participación hacia la constitución de la propia comunidad como sujeto activo de transformación de sus realidades, generando condiciones propicias para la toma de decisiones autónoma y conjunta sobre el propio proceso de salud-enfermedad-cuidados. Este proceso debe estimular la reflexión crítica y la capacidad de intervención y de co-gestión de los problemas sociales por parte de los individuos y colectivos. (Organización Panamericana de la Salud, 2014).

Con base a esos conceptos, el proyecto extensionista es para la comunidad educativa un espacio de participación, cultural, artístico y comunicativo. (Sanguineti; PEREYRA, 2016). El proyecto de producción de spot sonoros pretende buscar y atender la pluralidad social desde los valores de equidad, justicia, igualdad con sentido democrático. Permite a la Universidad unir espacios donde se desarrolla la propuesta de trabajo en terreno y se realiza un intercambio de saberes. (Pereyra, 2015a).

La producción de spot sonoros desde la universidad comprende además de la integración de estudiantes en la comunidad, una instancia del proceso-enseñanza/aprendizaje con la puesta en práctica de acciones que aportan al desarrollo social, el empoderamiento, la autonomía y los valores sociales a la hora de poder generar propuestas transformadoras.

La propuesta extensionista se desarrolló en el marco de las actividades prácticas de las cátedras participantes. El proyecto está dirigido a población cordobesa, quienes escucharan los spot difundidos por emisoras radiales adheridas al proyecto: 1.- Radio FM Sur. Emisora de Gestión comunitaria. Base territorial: Barrio Villa El Libertador, Ciudad de Córdoba. Destinatarios: adultos y adultos mayores de la zona Sur de la ciudad de Córdoba. Por la web, global. 2.- Radio Revés: Emisora de Gestión universitaria. Base 
territorial: Ciudad Universitaria, Ciudad de Córdoba. Destinatarios: jóvenes y adultos jóvenes de la ciudad de Córdoba. Por la web, global. 3.- Radio Eterogenia: Emisora de Gestión cultural. Base territorial: Barrio Centro, Ciudad de Córdoba. Destinatarios: jóvenes y adultos jóvenes. Sólo emite por la web, global.

\section{Metodología}

Los Objetivos propuestos fueron:

- Desarrollar un proyecto de extensión interdisciplinario-interuniversitario centrado en la promoción y prevención de salud mental en la ciudad de Córdoba.

- Contribuir con la sensibilización de las problemáticas de salud mental en estudiantes y hacia la comunidad.

- Brindar promoción y prevención de la salud mental mediante el uso de herramientas comunicacionales.

El proyecto se planificó en siete etapas que se desarrollaron en forma simultánea al dictado de las respectivas materias de cada unidad académica. Las mismas fueron de metodología participativa, con una guía de trabajo grupal con la finalidad de obtener material para la producción y realización de piezas comunicativas sonoras que promuevan la salud. Se dividió en siete etapas:

Primera: conformación de los grupos y modalidad de trabajo, selección del tema de interés. Se inició el encuentro grupal utilizando dinámicas de acercamiento y conocimiento inter-grupo. Se asignó tutores en cada equipo: con la función de guiar en la búsqueda bibliográfica de contenidos centrales según el tema de interés de cada grupo y que tuviera relevancia como problemática dentro de la comunidad.

Segunda: investigación bibliográfica sobre la temática, realización de entrevistas y presentación de informe escrito como requisito de las materias (debía contener introducción, marco teórico, referencias bibliográficas). El monitoreo del trabajo bibliográfico estuvo a cargo de los tutores de la Cátedra de Salud Mental y Psiquiatría dado la especificidad del contenido.

Tercera: definición de contenidos y estrategia comunicativa del audio, con el acompañamiento de los tutores de las distintas unidades académicas.

Cuarta: escritura del guion: Los docentes de la cátedra de producción radiofónica de F.C.I. brindaron su aporte dada la experiencia en producción y realización de anteriores campañas radiofónicas.

Quinta: grabación y edición de los spot en dos estudios de grabación puestos a disposición de la experiencia (estudios de HI-FI y Gabinetes de FCI).

Sexta: socialización y cierre de la producción sonora a la comunidad educativa inter cátedras, difusión de audios en la universidad.

Séptima: inclusión de los spot para su difusión en radios comunitarias de la ciudad de Córdoba. Las vivencias y experiencias de los docentes con los estudiantes durante el inicio, desarrollo y cierre se plasmaron en fotografías difundidas solo en el aula virtual y redes sociales de la diferentes Cátedras habilitadas.

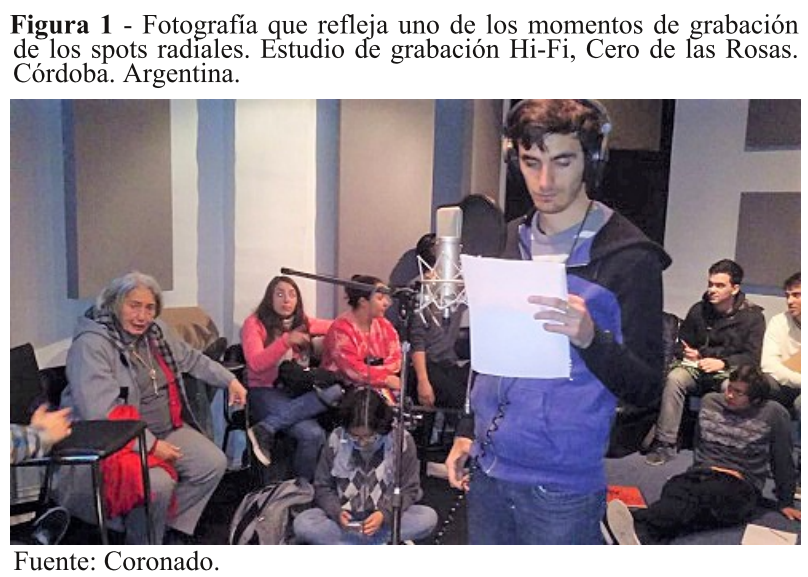

\section{Resultados}

Participaron de la experiencia doscientos alumnos por la cátedra de Enfermería en Salud Mental y Psiquiatría, Escuela de Enfermería, cuarenta y nueve de la cátedra de Producción Radial de la Carrera de Publicidad, Universidad Siglo 21 y veinte estudiantes por la cátedra de Producción Radiofónica de la Facultad de Ciencias de la Comunicación. Los mismos se constituyeron en cuarenta equipos de trabajo de seis integrantes cada uno, que representaban a las tres unidades académicas para desarrollar la tarea. Se cumplimentaron las sietes etapas propuestas en el proyecto, logrando todos los grupos cumplir con el objetivo del proyecto extensionista, produciéndose 40 spot sonoros. De los cuales el $57 \%$ refirieron a la temática de promoción en salud mental (comunicación saludable, construcción de redes de apoyo, promoción de factores protectores, habilidades sociales, entre otros) y el $43 \%$ restante fueron dedicados a la prevención de sufrimiento psíquico (alcoholismo, suicidio, ansiedad y estrés laboral). Todos ellos cumplieron el requisito de tener una duración menor a un minuto, dar un mensaje claro de salud mental acorde al marco teórico consultado y con lenguaje coloquial que tuviera en cuenta la población destinataria y sus raíces culturales. (Fábregas et al., 2018).

Respecto al tiempo planificado para la producción de spot, se ajustó al cronograma establecido permitiendo ejecutar todas las acciones previstas. En cuanto al apoyo técnico pedagógico, se contó con la experiencia de tutores de proyecto y coordinadores conformado por los docentes de las cátedras intervinientes de Escuela de Enfermería, Cátedra de Salud Mental, Facultad de Ciencias de la Información, Cátedra de Producción Radiofónica y Universidad Siglo 21, Cátedra de Publicidad. Cada asignatura aportó sus saberes en los diferentes momentos del desarrollo del proyecto, como los principales problemas de salud mental de la población Cordobesa, sostenida por la Cátedra de Salud Mental, la estrategia comunicativa del audio, la grabación y edición las Cátedras de Producción Radiofónica y Socialización y cierre de la producción sonora a la 
comunidad por la Cátedra de Publicidad, para la cual se seleccionaron radios comunitarias donde se difundieron las grabaciones de los spots sonoros, dentro de las cuales se pueden mencionar la Ranchada, Barrio sin Plaza y Radio la Curva.

\section{Consideraciones finales}

Logros: se pudo visualizar la participación activa de familiares y amigos de los estudiantes, donde hubo fusión de conocimiento, debate y reflexión. Este espacio constituido colectivamente permitió el enriquecimiento de las relaciones sociales, incrementando los saberes entre estos sujetos sociales.

La actividad extensionista les otorgó a los estudiantes herramientas metodológicas de construcción participativa, conocimientos y aprendizajes socialmente útiles, que le permitió abordar aquellos contenidos construidos desde una posición crítica. (Pereyra, 2015c). A su vez permitió aportar al vínculo práctica-teoría, permitiendo arribar juntos (docentes y estudiantes) al reconocimiento real, en la comunidad de la problemática en el campo dela salud mental.

El efecto directo en los estudiantes fue: la sensibilización y difusión a la temática, cimentación de valores colectivos: la vida, la salud, la solidaridad, aceptación de nuevos roles y trabajo con el otros y el aprendizaje de saberes de actores sociales.

El impacto en los docentes: fortalecimiento de experiencias intercátedras e interdisciplinarias. Constitución de espacios de innovación pedagógica desde la integralidad del proceso educativo y afianzamiento de modelos educativos transformadores. (Moreno, 2015)

Se produjo un intercambio de saberes, experiencias, costumbres y historias personales que enriquecieron la experiencia. A su vez el carácter de transdisciplinariedad (BECERRA, 2014) otorgó a cada contenido curricular un singular enfoque, profundización e implicancia al coincidir en nudos temáticos como ser promoción de la salud y la comunicación.

Dificultades: La articulación entre los alumnos de las distintas unidades académicas para encontrar espacios de trabajos grupales, lograr acuerdos, tolerar $\mathrm{y}$ ceder, argumentar posiciones y equilibrar el esfuerzo.

\section{Conclusión}

En la puesta en marcha del proyecto de extensión universitaria "Promoción de la salud mental en producción de spots sonoros", interdisciplinariointeruniversitario centrado en la promoción de salud mental destinado a la población cordobesa, se logró la sensibilización de las problemáticas de salud mental en los estudiantes y en su entorno. La propuesta de innovación pedagógica generó transformación en las redes, con prácticas vinculares, integrales y participativas entre la comunidad educativa, los estudiantes, familiares y comunicadores (Boruchalski, 2017). Esa fusión desde el aula a la comunidad permitió apoderarse de conceptos tales como el empoderamiento, la autonomía y ciudadanía.

Esta experiencia de responsabilidad formativa permite a la Universidad devolver a la comunidad el conocimiento transformado en acción comunicativa en salud. (Sanguineti; Pereyra, 2015).

\section{Referências}

ARGENTINA. Poder Legislativo Nacional. Ley Nacional De Salud Mental $\mathbf{N}^{\mathbf{0}} \mathbf{2 6 6 5 7}$. Derecho a la Protección de la Salud Mental. Disposiciones complementarias. Derogase la Ley No 22.914.Sanción: 25/11/2010; Promulgación:

02/12/2010; Boletín Oficial. Buenos Aires. Argentina: 03 dez. 2010. Disponible

en: $<$ http://www.msal.gob.ar/saludmental/images/stories/info -equipos/pdf/ley-nacional-salud-mental-26.657.pdf $>$ Acceso en: 10 mayo 2019

ARGENTINA. Presidencia de la Nación. Defensoria del PÚBLICO. Texto Completo de la Ley $\mathbf{N}^{0} \mathbf{2 6 5 2 2}$. Servicios de Comunicación audiovisual. Buenos Aires. Argentina Disponible en:

http://servicios.infoleg.gob.ar/infolegInternet/anexos/155000 -159999/158649/norma.htm. Acceso en: 20 jun 2019.

ARGENTINA. PRESIDENCIA DE LA NACIÓN. MINISTERIO DE SALUD. ARGENTINA. PRESIDENCIA DE LA NACIÓN. DIRECCION DE SALUD MENTAL Y ADICCIONES. MINISTERIO DE SALUD. Recomendaciones para el tratamiento de temas de salud mental en los medios. Buenos Aires. Argentina. Disponible en:

http://www.msal.gob.ar/images/stories/bes/graficos/0000000 $479 \mathrm{cnt}$-recomendaciones-tratamiento-temas-salud-mentalmedios.pdf. Acceso en: 10 mayo 2019.

\section{AUTORIDAD FEDERAL DE SERVICIOS DE} COMUNICACIÓN AUDIOVISUAL. Tratamiento de las prácticas suicidas en los medios audiovisuales. Buenos Aires: AFSCA. Argentina 2010. Disponible en:

$<$ https://es.calameo.com/books/001632393bdcc3ec29d8b > . Acceso en: junio 2019

BANG, C. L. Community strategies in mental health promotion: constructing a conceptual framework for approaching complex psychosocial problems.

Psicoperspectivas, Valparaíso, Chile, v. 13, n. 2, p. 109-

120, mayo 2014a. Disponible en https://scielo.conicyt.cl/scielo.php?

script $=$ sci arttext\&pid $=$ S0718-

9242014000200011\&lng=es\&nrm=iso. Acceso en: 21 ago. 2019.

http://dx.doi.org/10.5027/psicoperspectivas-Vol13-Issue2fulltext-399.

BANG, C. L. Estrategias comunitarias en promoción de salud mental: Construyendo una trama conceptual para el abordaje de problemáticas psicosociales complejas.

Psicoperspectivas, Valaparaiso, Chile, v. 13, n. 2, p. 109120, 2014b. Disponible en: http://www.psicoperspectivas. $\mathrm{cl} / \mathrm{index} . \mathrm{php} / \mathrm{psicoperspectivas/article/view/399}$. Acceso en: 20 jun. 2019. 
BANG, C. L. Los profesionales de salud y salud mental en prácticas comunitarias: descubriendo una experiencia participativa de promoción de salud mental en red. Revista Facultad Nacional de Salud Pública, Medellín, v. 32, n. 1, p. S134-S142, 2014c. Disponible en: https://ri.conicet.gov.ar/handle/ 11336/46516 Acceso en: ago. 2019.

BECERRA, G. Interdisciplina y Sistemas Complejos: Un enfoque para abordar problemáticas sociales complejas. Revista de Investigación en Psicología Social, v.1, n.1, p. 34-43, 2014. Disponible en:

https://www.researchgate.net/publication/270798180 Interdi sciplina_y_sistemas_complejos_Un_enfo que para abordar problematicas sociales complejas: Acceso en: 20 ago. 2019.

BORUCHALSKI, E. Integración docencia y extensión: otra forma de aprender y de enseñar Reseña de libro. Revista Universidad Nacional del Litoral. Argentina. 2017.

Disponible en:

https://bibliotecavirtual.unl.edu.ar/publicaciones/index.php/ Extension/article/view/6379/9338>.Acceso en: 20 mar. 2019.

FÁBREGAS, M.; TAFUR, A.; GUILLÉN, A.; BOLAÑOS, L.; MÉNDES, J. L. ; FERNÁNDES DE SEVILLA, P. Guía de estilo sobre salud mental para medios de comunicación: las palabras sí importan. Madrid: Confederación Salud Mental España, 2018. Disponible en: https://www.consalud mental.org/publicaciones/Guia-estilosalud-mental.pdf. Acceso en: 21 ago. 2019

GUÍA para el tratamiento mediático responsable de la salud mental. Ciudad Autónoma de Buenos Aires. Argentina: Ed. Eudeba, 2014. Disponible en:

https://defensadelpublico.gob.ar/wp-content/

uploads/2016/08/guia_salud_mental_marzo2015-1.pdf $>$. Acceso en: 10 mar $2 \overline{019}$.

HENAO, Silvia et al. Políticas públicas vigentes de salud mental en Suramérica: un estado del arte. Rev. Fac. Nac. Salud Pública, Medellín, v. 34, n. 2, p. 175-183, Aug. 2016. Disponible en

http://www.scielo.org.co/scielo.php?script=sci arttext\&pid= S0120-386X2016000200007\&lng $=$ en $\& n \mathrm{rm}=\overline{\mathrm{is}}$. Acceso en: 21 ago. 2019.

MORENO, M. B. Las innovaciones pedagógicas y al gestión de la educación en la Escuela Nueva Laboratorio "Emma Gamboa" de la Universidad de Costa Rica y el
Instituto Educativo Moderno. Revista Gestión de la Educación, v.5, n.2, p. 39-68, 2015. Universidad de Costa Rica. San José, Costa Rica. Disponible en: https://revistas.ucr.ac.cr/index.php/gestedu/article/view/1993 9. Acceso en: ago. 2019

ORGANIZAÇION MUNDIAL SALUD. Centro de Prensa. Salud mental: fortalecer nuestra respuesta 30 mar. 2018. Disponible en: < https://www.who.int/es/news-room/factsheets/detail/mental-health-strengthening-our-response $>$. Acceso en: ago. 2019.

ORGANIZACIÓN PANAMERICANA DE LA SALUD. Plan de acción sobre salud mental 2015-2020. 2014. Disponible en: <www.paho.org/hq/dmdocuments/2015/plande-accion-SM-2014.pdf?ua=1> Acceso en: 20 mar. 2019.

PEREYRA, M. Actividades y experiencias radiofónicas en complementariedad entre estudiantes de la universidad y radios comunitarias. En: Foro de Extensión Universitaria, 5. Córdoba. Argentina. 2015a. Disponible en: https://rdu.unc.edu.ar/bitstream/

handle/11086/4637/sanguineti_pereyra_vocacion_radio.pdf? sequence $=6 \&$ is Allowed $=y$. Acceso en:: Jul. $201 \overline{9}$

\section{PEREYRA, M. En campaña, Interdisciplina para la}

Salud Mental: proyecto extensionista.Córdoba. Argentina. 2015b. Disponible en:

https://www.youtube.com/watch? $\mathrm{v}=\mathrm{pKG}$ - oTnNFfo $\& \mathrm{t}=1 \mathrm{~s}$. Acceso en:. 20 jun 2019.

PEREYRA, M. Extender las aulas, un proyecto de articulación de docencia, investigación y extensión desde la producción radiofónica. En: Congreso de la Red de Carreras de Comunicación Social y Periodismo de Argentina, Actas...17, 25 y 26 agosto 2015. Disponible en: http://www.redcom2015.eci.unc.edu.ar/files/REDCOM/EJE 5/redcom_-5_-_13_Pereyra\%20Quinteros.pdf $>$. Acceso en: 17 abr. $2 \overline{01} \overline{9}$

SANGUINETI, S.; PEREYRA, M. (Comp.).Vocación de radio: procesos de producción. Córdoba, Argentina: Ed. Brujas, 2015. Disponible en: < https://rdu.unc.edu.ar/handle/11086/4637> Acceso en: 20 mar. 2019:

VAZQUEZ, J. "El derecho a vivir y a ser incluido en la comunidad". En: Conferencia Regional de Salud Mental. Actas... Santiago de Chile: OPS/OMS, 2015. 